\title{
A NEW ROBOTIC MANIPULATOR IN CONSTRUCTION BASED ON MAN-ROBOT COOPERATION WORK
}

Toshio Fukuda, Yoshio Fujisawa, Fumihito Arai

Dept. of Mechanical Engineering, Nagoya University

Furo-chyo, Chikusa-ku

Nagoya, 464-01, Japan

Eiji Muro, Haruo Hoshino, Kenji Miyazaki, Tadao Mikami

Takenaka Corporation, Technical Research Laboratory

Kazuhiko Ohtsubo, Kazuo Uehara

Komatsu Ltd., Technical Research Center

\section{SUMMARY}

This paper deals with the mechanism and the control method of a new robotic manipulator in construction based on man-robot cooperation work. Because of the coming labor shortage of the aging society in the near future, robots will be used in construction fields more and more. To support robot operation, this study aims to develop a cooperation work type of robot to be controlled easily by the direct teaching. The robot has the following features: (1) It has 4 D.O.F. and a parallel link mechanism. (2) It has a operation control sensor and a force control sensor to cooperate with the human operator and to control the contact force between robot and object. (3) The end effector of this robot has a vacuum system to carry payloads. With these features, this robotic system makes it possible to carry heavy payloads easily and this system can be used for various kinds of tasks.

Key Words: Man-Robot Cooperation, Direct Teaching, Robotic

\section{INTRODUCTION}

The labor shortage in construction and civil engineering fields is the serious problem in the world. The tasks required at these fields are so hard that many workers may counter labor disasters. In order to settle these problems and improve the working efficiency and safety, the applications of robots to these fields are required all over the world. The conventional robots which are used now are moved by the remote operation or the teaching. But the work which is done in construction and civil engineering fields have various kind of tasks. For the present robots, the perfect automation in construction and civil engineering fields may be awfully difficult. And if it is possible, the system will be complicated.

Then, the authors have proposed A NEW CONSTRUCTION MANIPULATOR BASED ON MAN-ROBOT COOPERATION WORK/1,2/. 
By including the judgments of human operator in the robotic system, we can come to meet with the various tasks $/ 3,4 /$.

This robot has two force sensors. One is used for force control, while the other is for operation control. By using these two sensors, the human operator can finish various tasks by cooperating with the robot.

In this paper, we explain the mechanism and the control method of a new robotic manipulator in construction based on man-robot cooperation work.

\section{MECHANISM OF THE ROBOT}

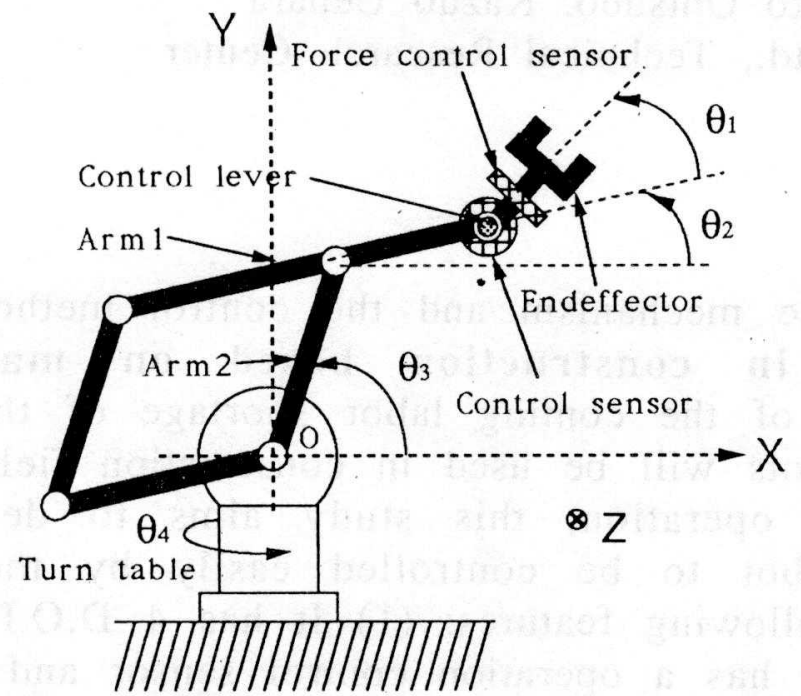

Fig. 1 Shape of Robot

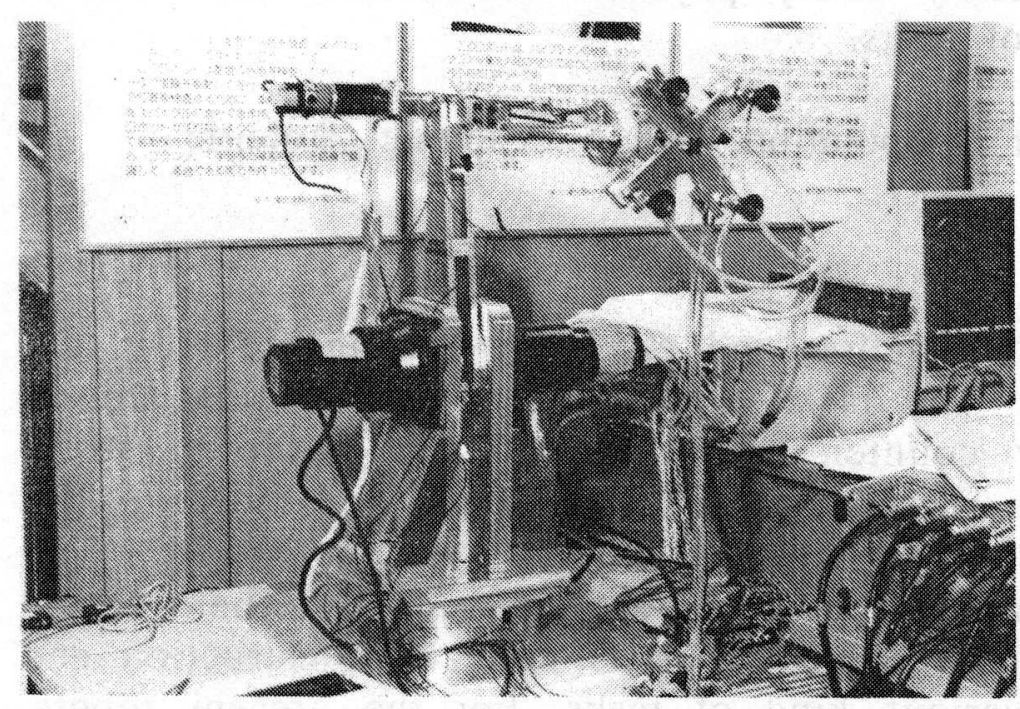

Fig. 2 Photograph of Robot
The robot which we made and used in this experiments has the parallel link mechanism, as shown in Figs.1 and 2. By using this mechanism, we can mount actuators on pedestal. As a result, we can make the arm light. This robot has 4 degrees of freedom and we used four DC motors as actuators. The detail figure of this end effector is shown in Fig. 3. The end effector has a vacuum system and the operation control sensor lever to carry payloads.

This robot has two force sensors. One is named the force control sensor(FCS), while the other is named the operation control sensor $(O C S)$. OCS can measure the operation control force applied by the human operator to this lever, and is attached to the tip of the first arm. FCS can measure the contact force between the robot and the objects, and is attached to the location between the end effector and the first arm. By using the OCS, we can move this manipulator to the direction in which we want to move. By using the FCS, we can control the contact force, as a result, we can avoid applying excess forces against objects and breaking the manipulator and the objects.

The movable area is shown in Fig. 4. The movable rotation angle of pedestal extends from $-125 \mathrm{deg}$. to $125 \mathrm{deg}$. 


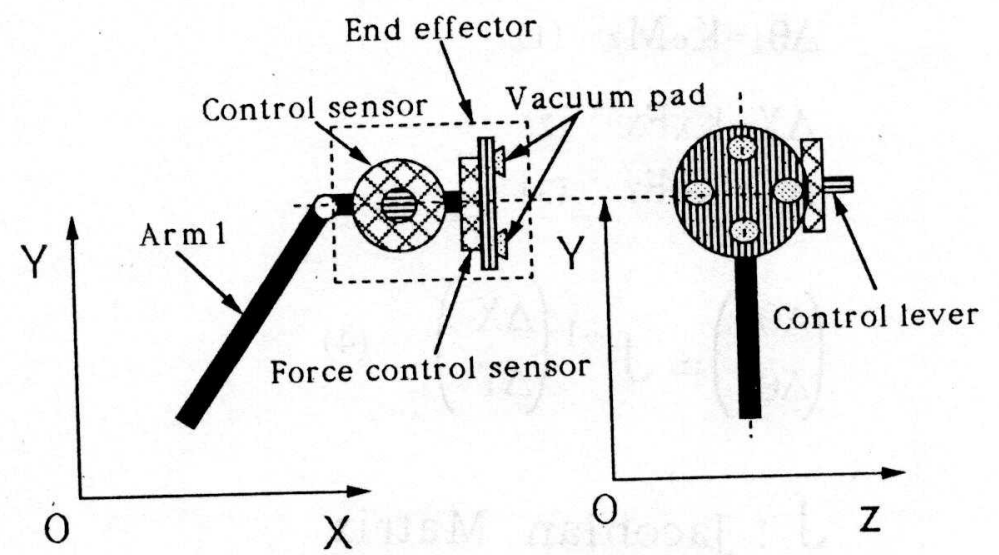

Fig. 3 Detail Figure of End Effector

A :Tip position of Arm 1

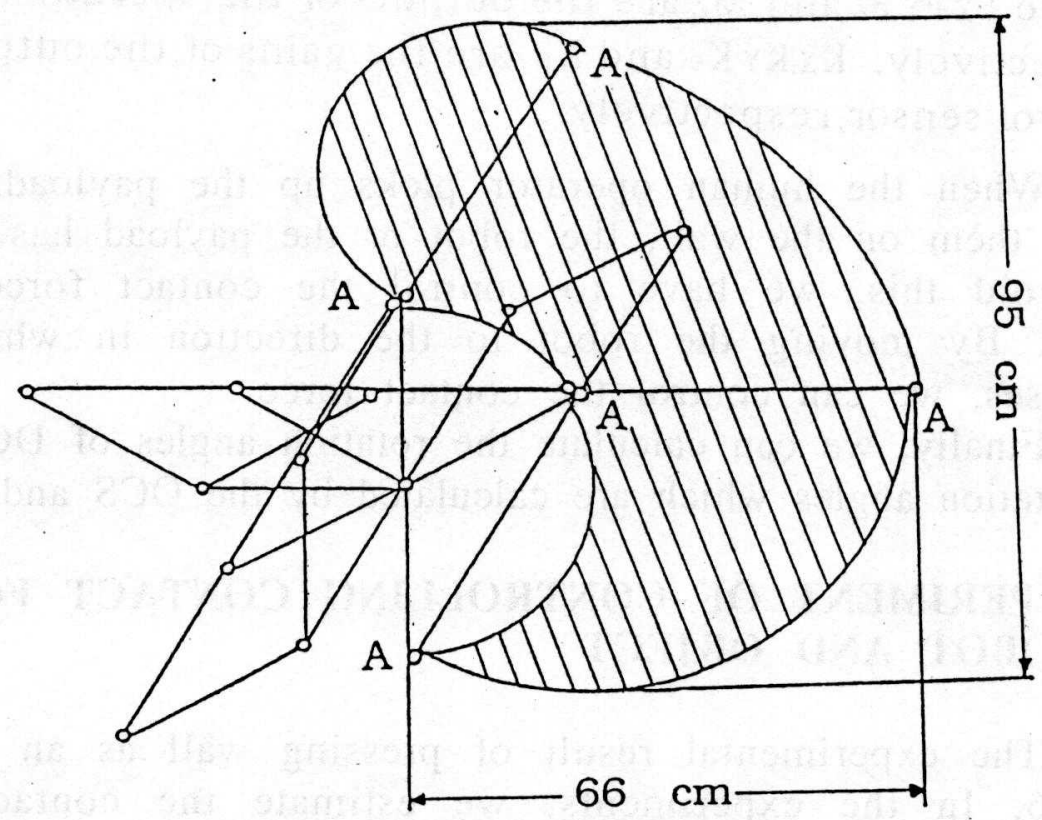

Fig. 4 Movable Area

\section{CONTROL METHOD OF THE ROBOT}

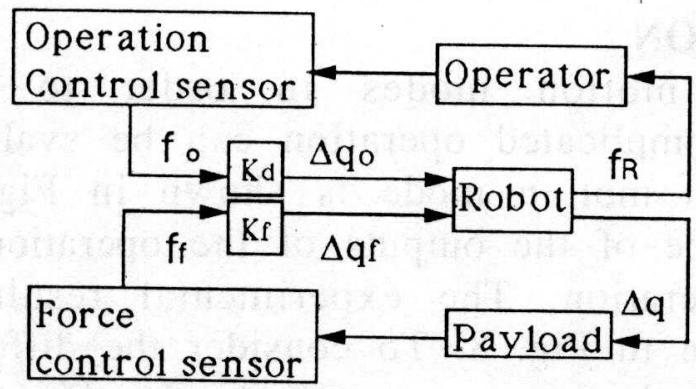

Fig. 5 Control System
The control system of this robot is shown in Fig. 5. The signal from the FCS and the signal from the OCS are used to control this robot. According to the measured force by the FCS and the OCS, the computer calculates the displacement of this robot and the rotation angle of each DC motor. In sampling time, we have to rotate each 
DC motors. The method of calculating the displacement and the rotation angle of each DC motor is described as follows:

$$
\begin{aligned}
& \Delta \theta_{1}=\mathrm{KeMz}_{\mathrm{e}} \\
& \Delta \mathrm{X}=\mathrm{KxFx} \\
& \Delta \mathrm{Y}=\mathrm{KyFY} \\
& \left(\begin{array}{l}
\Delta \theta_{2} \\
\Delta \theta_{3}
\end{array}\right)=\mathrm{J}^{-1}\left(\begin{array}{l}
\Delta \mathrm{X} \\
\Delta \mathrm{Y}
\end{array}\right) \\
& \mathrm{J}: \text { Jacobian Matrix } \\
& \Delta \theta_{4}=\mathrm{K}_{\mathrm{p}} \mathrm{F}_{\mathrm{z}}
\end{aligned}
$$

where FX,Fy,Fz and $\mathrm{Mz}$ are the outputs of the operation control sensor, respectively. $\mathrm{Kx}, \mathrm{KY}, \mathrm{Ke}$ and $\mathrm{Kp}$ are the gains of the outputs of the operation control sensor, respectively.

When the human operator picks up the payloads from the floor and places them on the wall, the robot or the payload has chance to be broken. To avoid this, we have to control the contact force between robot and object. By moving the robot to the direction in which the contact force decreases, we can control the contact force.

Finally, we can calculate the rotation angles of DC motors, according to the rotation angles which are calculated by the OCS and FCS.

\section{EXPERIMENT OF CONTROLLING CONTACT FORCE BETWEEN ROBOT AND OBJECT}

The experimental result of pressing wall as an example is shown in Fig. 6. In the experiments, we estimate the contact force, that is, we compare the contact force when the FCS is used with the contact force when the FCS is not used. As shown in Fig. 6, by using the FCS, we can control contact force, so that, we can avoid breaking the manipulator or the objects.

\section{EVALUATION OF OPERATION}

We set four fundamental motion modes in order to evaluate operation. We consider that any complicated operation can be evaluated by these four modes. The fundamental motion mode is shown in Fig. 8. We used the work time and the variance of the outputs of the operation control sensor so as to evaluate the operation. The experimental result of the evaluation of the operation is shown in Fig. 8. To consider the difference of the operator, the two operators did the same experiments. In this paper, the mode 2 is shown as an example. Here, we evaluate that the operation is 

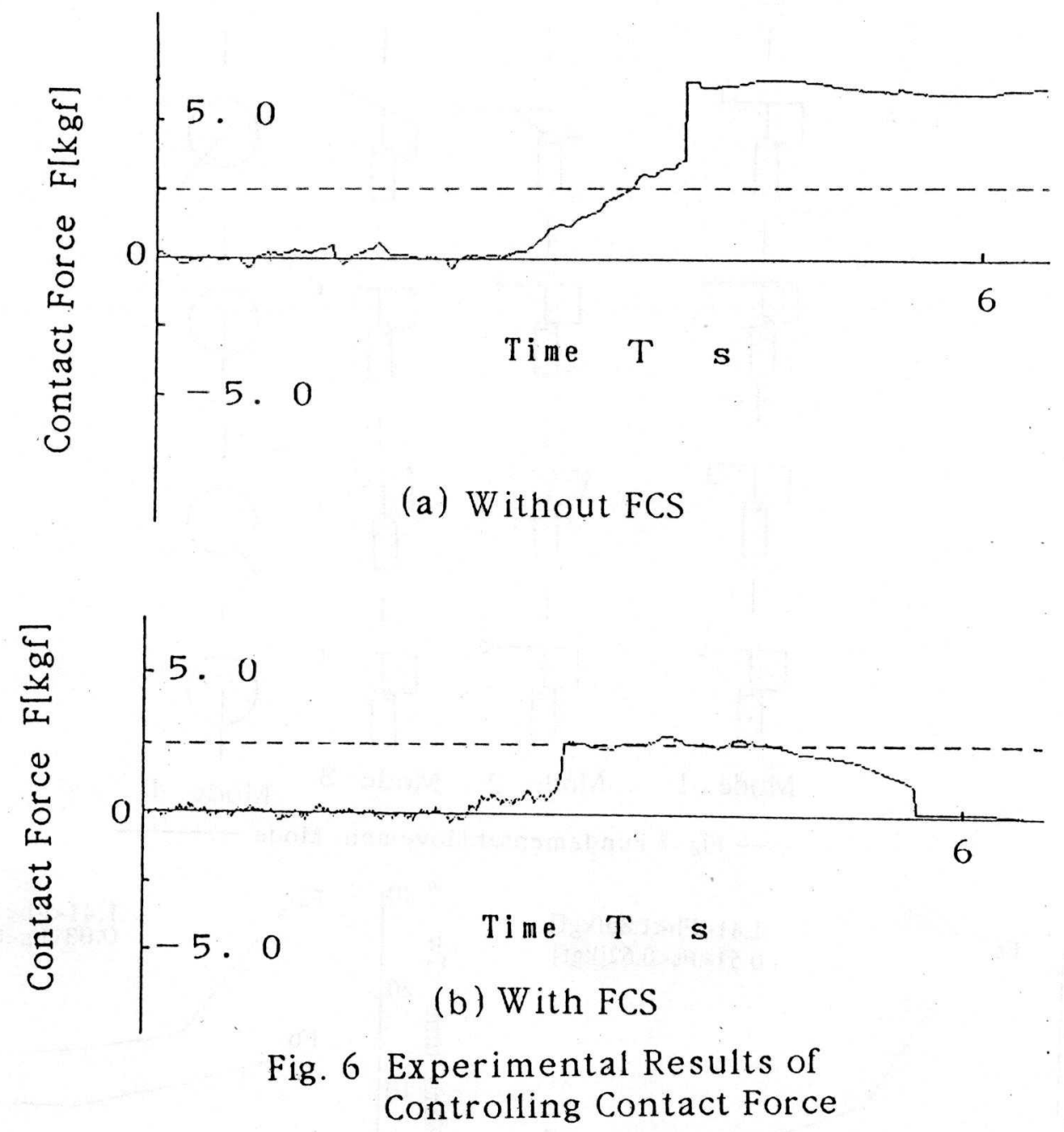

better when the work time is short and the variance is small.

From the experimental results shown in Figs. 8 and 9, the work time is long and the variance is small when the feedback gain of the output of the operation control sensor is small. On the other hand, the work time is long and the variance is big when the feedback gain is big. When we set gain larger, the work time tends to be long. There are subtle differences between the two operators, however the two results are same in general.

\section{CONCLUSION}

We proposed a new type robotic manipulator in construction based on man-robot cooperation work. By using the operation control sensor and the force control sensor, we can handle various works and control the contact force between the robot and the objects. We set four modes to evaluate the 


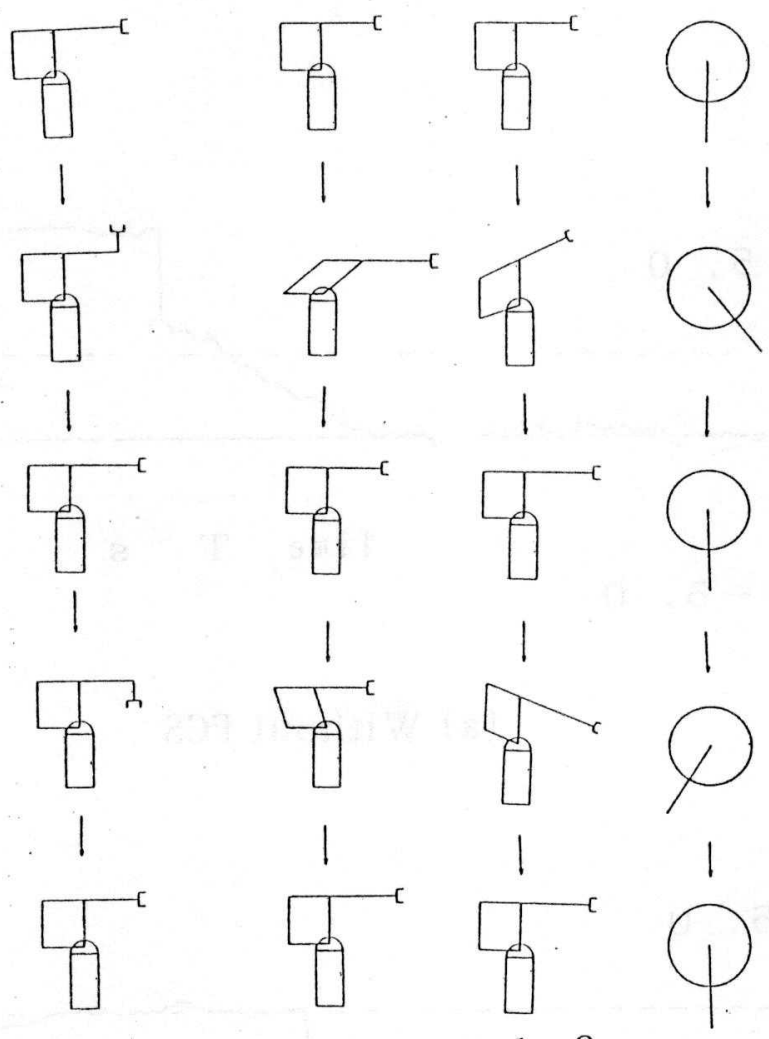

Mode 1 Mode 2 Mode 3 Mode 4

_ Fig. 7 Fundamental Movement Mode
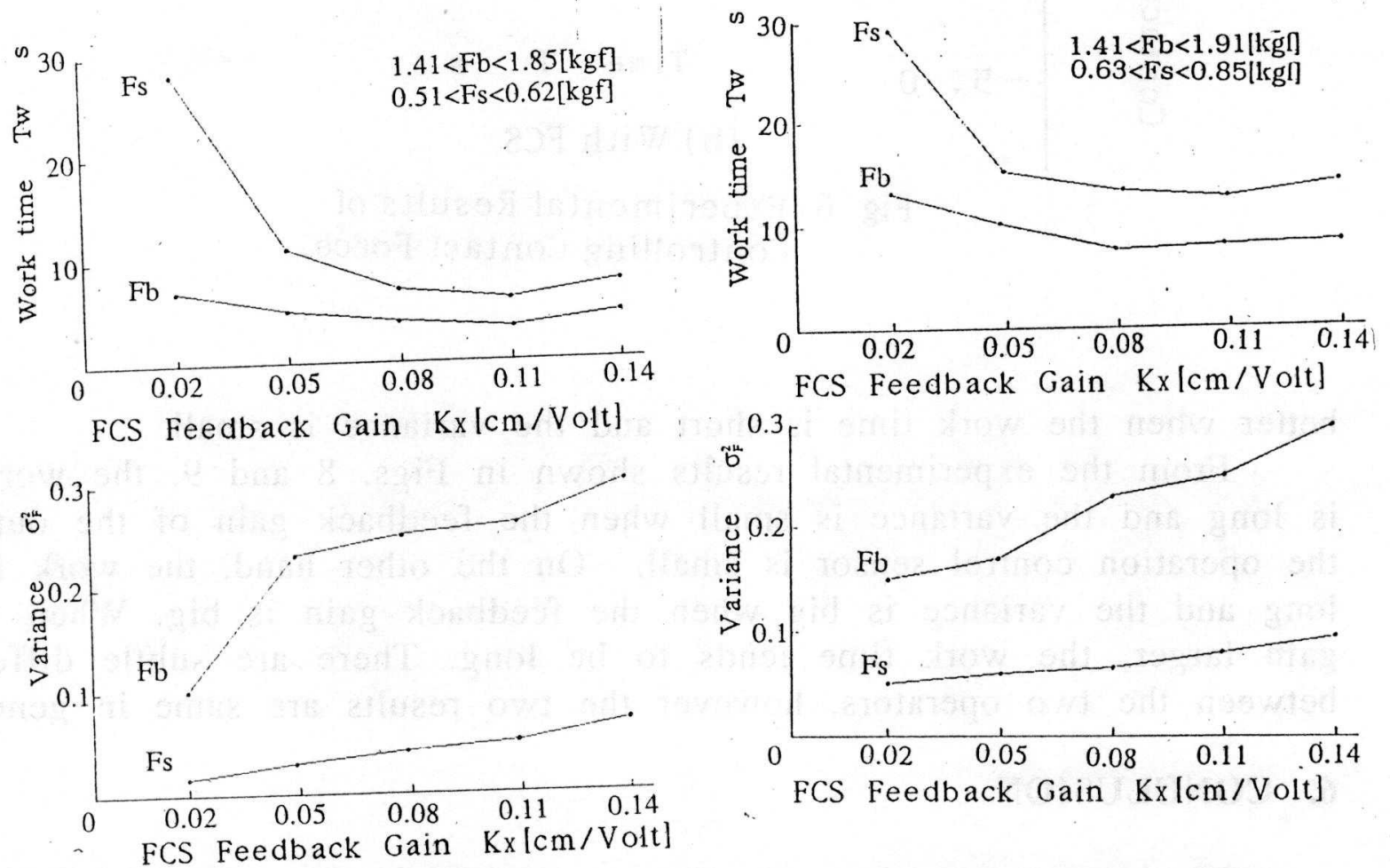

Fig. 8 Estimation of Operation(Subject A)

Fig. 9 Estimation of Operation(Subject B) 
operation, so that it can clarify through the experiments that there are some appropriate control gains for man-robot cooperation works.

\section{REFERENCES}

(1) T. Fukuda, et al, "A Control Method of Man-Robot Cooperation Work Type of Construction Manipulators", The First Construction Robot Symposium, pp. 85-92,1990(in Japanese).

(2) T. Fukuda, Y. Fujisawa, F. Arai, "Study on Man-Robot Cooperation Type of Manipulators(1st report, Mechanism and Control of Robotic Manipulator in Construction)", 8th Symposium of the Robotics Society of Japan, pp. 655656, 1990.

(3) Kazerooni, H, "Human Machine Interaction via the Transfer of Power and Information Signals", IEEE Intrenational Conference on Robotics and Automation, May 1989, Scottsdale, Arizona,pp. 1632-1642

(4) Kazerooni, H, et al, "Robust Compliant Motion for Manipulators", IEEE J. of Robotics and Automation, Vol.2, No.2, June 1986. 